\title{
Splenic artery pseudoaneurysm due to seatbelt injury in a glucose-6-phosphate dehydrogenase-deficient adult
}

\author{
Yu Zhen $\underline{\operatorname{Lau}}^{1}$, Ms, Yuk Fai $\underline{\operatorname{Lau}}^{1}$, Ms, Kang Yiu $\underline{\operatorname{Lai}}^{2}$, MBBs, Chu Pak $\underline{\operatorname{Lau}}^{3}$, MD, FRCP
}

\begin{abstract}
A 23-year-old man presented with abdominal pain after suffering blunt trauma caused by a seatbelt injury. His low platelet count of $137 \times 10^{9} / \mathrm{L}$ was initially attributed to trauma and his underlying hypersplenism due to glucose-6-phosphate dehydrogenase (G6PD) deficiency. Despite conservative management, his platelet count remained persistently reduced even after his haemoglobin and clotting abnormalities were stabilised. After a week, follow-up imaging revealed an incidental finding of a pseudoaneurysm (measuring $9 \mathrm{~mm} \times 8 \mathrm{~mm} \times 10 \mathrm{~mm}$ ) adjacent to a splenic laceration. The pseudoaneurysm was successfully closed via transcatheter glue embolisation; $20 \%$ of the spleen was also embolised. A week later, the platelet count normalised, and the patient was subsequently discharged. This case highlights the pitfalls in the detection of a delayed occurrence of splenic artery pseudoaneurysm after blunt injury via routine delayed phase computed tomography. While splenomegaly in G6PD may be a predisposing factor for injury, a low platelet count should arouse suspicion of internal haemorrhage rather than hypersplenism.
\end{abstract}

Keywords: G6PD, hypersplenism, splenomegaly, transcatheter embolisation, thrombocytopenia

\section{INTRODUCTION}

Visceral artery aneurysms are rare occurrences. One of the most common sites of arterial aneurysms is the splenic artery. ${ }^{(1)}$ True splenic artery aneurysms are uncommon, and pseudoaneurysms are even rarer. ${ }^{(2)}$ Herein, we describe a case of delayed splenic artery pseudoaneurysm due to a seatbelt injury in a glucose-6-phosphate dehydrogenase (G6PD)deficient adult, who was successfully treated via transcatheter embolisation.

\section{CASE REPORT}

A 23-year-old Chinese man with asymptomatic G6PD deficiency, presented with abdominal pain after suffering blunt trauma caused by a seatbelt injury. Subsequent imaging revealed the presence of mild splenomegaly with a splenic volume of $308 \mathrm{~mL}$ (splenomegaly is defined as splenic volume $>300 \mathrm{~mL}){ }^{(3)}$ and the patient's platelet count was on the lower side of normal. His low platelet count of $137 \times 10^{9} / \mathrm{L}$ at admission was initially attributed to trauma and his underlying hypersplenism due to glucose-6-phosphate dehydrogenase (G6PD) deficiency. The patient presented with classic seatbelt injury signs after a road traffic accident: neck pain and left upper quadrant tenderness following blunt trauma to the shoulder and abdomen. Upon admission, he was haemodynamically stable with no neurological deficits. Radiography of the chest, cervical spine and pelvis were unremarkable. Initial computed tomography (CT) of the abdomen revealed haemoperitoneum, and lacerations of the spleen, the liver (in segment 5), and at the medial aspect of the lower pole of the left kidney with significant perinephric haematoma. Imaging showed the presence of a small pneumothorax at the right hemithorax and left lung apex, but no pleural effusion. A gas pocket was detected at the right paraoesophageal region close to the gastro-oesophageal junction. An oesophageal rupture was excluded by subsequent gastrograffin swallow. Repeat CT of the abdomen on the following day demonstrated an increase in haemoperitoneum, but there was no change in the lacerations of the spleen, liver and left renal parenchyma. On delayed phase CT, there was also no gross pooling of contrast to suggest extravasation. It was agreed that the patient would be managed conservatively. The patient had considerable haemodilution, with haemoglobin level reduced from $15.0 \mathrm{~g} / \mathrm{dL}$ to $11.0 \mathrm{~g} / \mathrm{dL}$ (Table I) within five days of admission.

The patient's renal function was normal. There was a transient elevation of transaminase, which resolved completely. The mild thrombocytopenia observed in the patient since admission was attributed to hypersplenism in view of his G6PD deficiency. Although the patient was managed conservatively, his platelet count was persistently reduced despite stabilisation of his haemoglobin and clotting abnormalities; the patient's platelet count continued to fall to $99 \times 10^{9} / \mathrm{L}$. Both prothrombin and activated thromboplastin times were normal, but there was an increase in D-dimer level from 2,896 ng/mL to $3,192 \mathrm{ng} / \mathrm{mL}$ (the normal limit is $\leq 500 \mathrm{ng} / \mathrm{mL}$ ) one week after admission. Follow-up CT showed no changes in the lacerations of the spleen, liver and kidney, and revealed a resolving haemoperitoneum. However, this highlighted a

\footnotetext{
${ }^{1}$ King's College London School of Medicine, London, United Kingdom, ${ }^{2}$ Intensive Care Unit, Queen Elizabeth Hospital, ${ }^{2}$ Division of Cardiology, Department of Medicine, University of Hong Kong, Hong Kong SAR, China

Correspondence: Prof Chu Pak Lau, Director, Cardiac Heart Health Centre, Suite 1303, Central Building, 3 Pedder Street, Central, Hong Kong, China. cplau@hkucc.hku.hk
} 
Table I. Haematological changes of the patient.

\begin{tabular}{lcccccc}
\hline Parameter & $\begin{array}{c}\mathbf{1} \text { yr before } \\
\text { admission }\end{array}$ & $\begin{array}{c}\text { At } \\
\text { admission }\end{array}$ & $\begin{array}{c}\text { Before } \\
\text { embolisation }\end{array}$ & $\begin{array}{c}\text { Post } \\
\text { embolisation }\end{array}$ & $\begin{array}{c}\text { At } \\
\text { discharge }\end{array}$ & $\begin{array}{c}\mathbf{4} \text { wks post } \\
\text { embolisation }\end{array}$ \\
\hline Haemoglobin $(\mathrm{g} / \mathrm{dL})$ & 14.8 & 15.0 & 11.0 & 11.3 & 11.6 & 14.8 \\
White blood cell $\left(\times 10^{9} / \mathrm{L}\right)$ & 4.7 & 10.5 & 5.3 & 7.6 & 4.0 & 4.0 \\
Platelet $\left(\times 10^{9} / \mathrm{L}\right)$ & 148 & 137 & 99 & 134 & 175 & 131 \\
\hline
\end{tabular}

The normal ranges for haemoglobin, white blood cell, and platelet count are $13.4-17.0 \mathrm{~g} / \mathrm{dL}, 3.7-9.2 \times 10^{9} / \mathrm{L}$, and $145-370 \times 10^{9} / \mathrm{L}$, respectively.

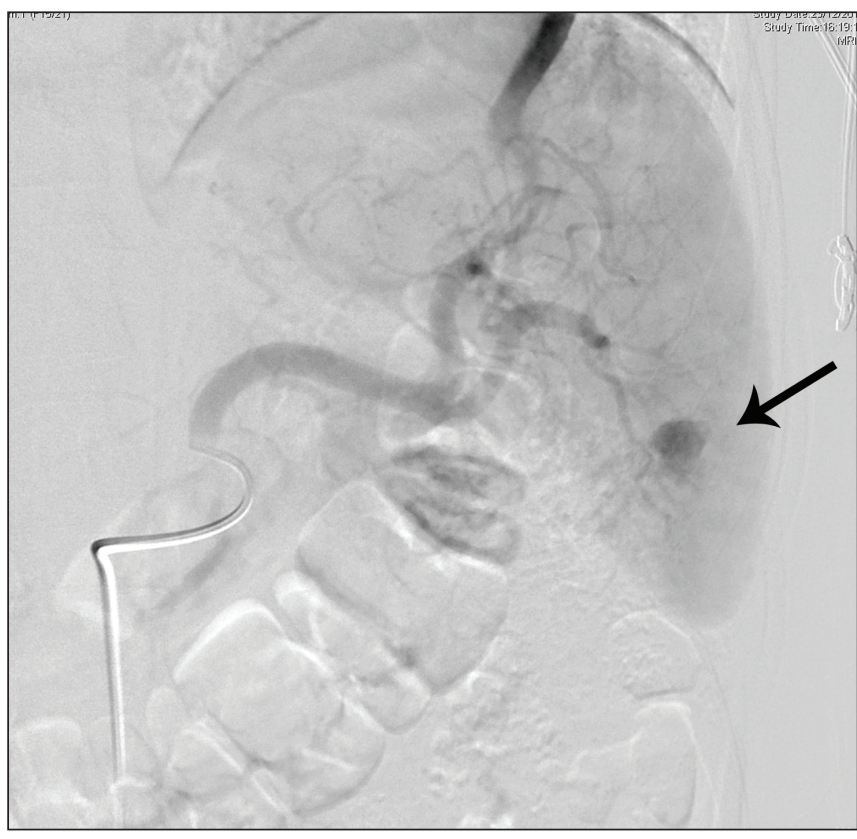

Fig. 1 Selective angiogram of the splenic artery shows a 1-cm large splenic artery pseudoaneurysm in the inferior branch of the splenic artery (arrow).

newly developed contrast-enhancing, arterial-phase lesion in the spleen, which was adjacent to the splenic laceration, raising suspicion of a pseudoaneurysm or arteriovenous fistula. The patient then underwent Doppler ultrasonography, revealing a hypoechoic nodule measuring $9 \mathrm{~mm} \times 8 \mathrm{~mm} \times 10 \mathrm{~mm}$ close to the splenic hilum. A $3 \mathrm{~F}$ microcatheter was used to selectively catheterise the distal splenic artery supplying the pseudoaneurysm (Fig. 1). The pseudoaneurysm was then closed via transcatheter glue embolisation with $0.5 \mathrm{~mL}$ of $30 \%$ glue. Post-embolisation angiography showed satisfactory occlusion of the pseudoaneurysm, with embolisation of about $20 \%$ of the surrounding inferior portion of the spleen (Fig. 2). Thrombocytopenia in the patient was normalised after embolisation, but his platelet count was higher at discharge than that at his premorbid state (Table I). The patient was discharged with no complications seven days after the procedure, and repeat imaging performed four weeks after embolisation showed resolution of the haematoma. The platelet count subsequently reduced to the patient's premorbid platelet level.

\section{DISCUSSION}

This case highlights the delayed occurrence of splenic artery pseudoaneurysm and the pitfalls in rendering the diagnosis. Despite an apparently stable haemoglobin level

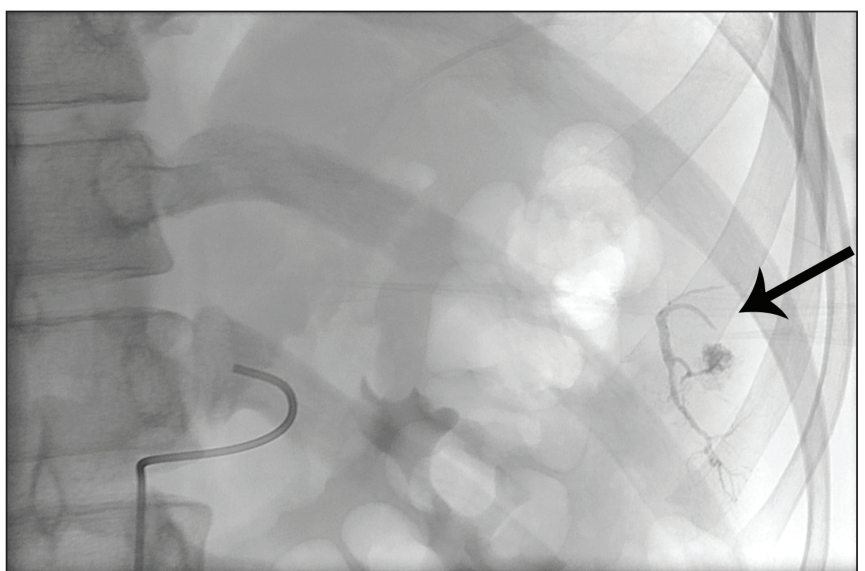

Fig. 2 Complete occlusion of the pseudoaneurysm after $0.5 \mathrm{~mL}$ of $30 \%$ glue embolisation, with residual contrast in the embolised artery (arrow).

and haemodynamic condition, delayed phase $\mathrm{CT}$ is essential in the exclusion of this condition. Continued decreases in platelet count with an elevated D-dimer level suggested that the low platelet count was due to continued clot formation instead of hypersplenism. While G6PD deficiency may result in an enlarged spleen, which is therefore liable to traumatic injury, it should not be used to explain thrombocytopenia in the trauma setting.

The spleen is the solid organ most frequently injured in blunt abdominal trauma, which could later lead to an intraabdominal injury. ${ }^{(4,5)}$ Splenic artery pseudoaneurysms are usually caused by pancreatitis, trauma, and rarely, peptic ulcer disease. ${ }^{(2)}$ It has been shown that $2.5 \%$ of splenic artery pseudoaneurysms can present incidentally, as in this case.(2) The classical clinical presentations include abdominal pain $(29.5 \%)$, haematochezia or melaena $(26.2 \%)$, haemorrhage into the pancreatic duct $(20.3 \%)$ and haematemesis $(14.8 \%)^{(2)}$ Strikingly, a relatively high risk of splenic artery pseudoaneurysm rupture (37\%) has been found, and if left untreated, the mortality rate is approximately $90 \% .^{(6,7)}$ The size of the pseudoaneurysm does not determine the chance of spontaneous rupture; the smallest and largest pseudoaneurysms reported were $0.3 \mathrm{~cm}$ and $17 \mathrm{~cm}$ in diameter, respectively, and both were ruptured at presentation. ${ }^{(2)}$ Therefore, despite the available option of conservative management, the repair of all splenic artery pseudoaneurysms (of various sizes, and with or without symptoms) should be performed to prevent possible spontaneous rupture. ${ }^{(8)}$

Transcatheter embolisation is a recent nonsurgical procedure in the management of splenic artery pseudoaneurysms. ${ }^{(9)}$ 
Despite a lower success rate of $85 \%$ compared to typical surgical intervention, transcatheter embolisation carries lower rates of mortality and morbidity. ${ }^{(10)}$ Independent of the type of intervention, recognition and early diagnosis of splenic arterial pseudoaneurysm form the cornerstone of treatment. Hence, it is vital to carry out further follow-up investigations such as $\mathrm{CT}$, Doppler ultrasonography and angiography to rule out splenic artery pseudoaneurysm at the earliest possible stage.

G6PD deficiency is an X-linked recessive hereditary disorder that has a higher incidence in males than females. It is caused by mutations in the G6PD gene, and is the most common enzymatic disorder that involves red blood cells in humans. ${ }^{(11)}$ Approximately 400 million people worldwide are affected by this disorder. ${ }^{(12)}$ The G6PD enzyme is a catalyst in the pentose phosphate pathway, which produces antioxidants that protect cells against oxidative damage. ${ }^{(13)}$ Therefore, a G6PD-deficient individual has a high risk of developing a haemolytic crisis when exposed to oxidative stressors, with chronic haemolysis being reported in some mutations. ${ }^{(13,14)}$ Splenic enlargement may result from haemolysis, although studies on splenic sizes using abdominal ultrasonography are controversial. ${ }^{(14)}$ In our patient, the spleen was enlarged and his platelet count was already on the low side of normal before the traumatic incident. His splenomegaly may have predisposed him to splenic laceration. His haemoglobin and platelet count decreased further during hospitalisation without any use of antioxidant medications, which suggested the presence of internal bleeding rather than haemolysis. Of interest, the patient's platelet count at discharge was higher than his premorbid platelet level, suggesting that the 'transcatheter partial splenectomy' performed could have reduced haemolysis in a manner similar to splenectomy. ${ }^{(14)}$ As splenic regeneration commenced, his platelet count was reduced to that of his premorbid state.

To the best of our knowledge, this is the first reported case of splenic artery pseudoaneurysm due to a seatbelt injury in a G6PD-deficient adult with progressive thrombo- cytopenia, which was successfully managed via transcatheter embolisation. A high index of suspicion and routine CT were needed to arrive at the diagnosis. G6PD deficiency may cause splenic enlargement and a mild decrease in platelet count, but a low platelet count in the presence of trauma should not be attributed to hypersplenism alone. Instead, continued haemorrhage should be suspected.

\section{REFERENCES}

1. Baker KS, Tisnado J, Cho SR, Beachley MC. Splanchnic artery aneurysms and pseudoaneurysms: transcatheter embolization. Radiology 1987; 163:135-9.

2. Tessier DJ, Stone WM, Fowl RJ, et al. Clinical features and management of splenic artery pseudoaneurysm: case series and cumulative review of literature. J Vasc Surg 2003; 38:969-74.

3. Wu WC, Chiou YY, Hung HH, et al. Prognostic significance of computed tomography scan-derived splenic volume in hepatocellular carcinoma treated with radiofrequency ablation. J Clin Gastroenterol 2012; 46:789-95

4. Schafermeyer R. Pediatric trauma. Emerg Med Clin North Am 1993; 11:187-205.

5. Powell M, Courcoulas A, Gardner M, et al. Management of blunt splenic trauma: significant differences between adults and children. Surgery 1997; 122:654-60.

6. Huang IH, Zuckerman DA, Matthews JB. Occlusion of a giant splenic artery pseudoaneurysm with percutaneous thrombin-collagen injection. J Vasc Surg 2004; 40:574-7.

7. LiPuma JP, Sachs PB, Sands MJ, Stuhlmiller S, Herbener TE. Angiography/ interventional case of the day: splenic artery pseudoaneurysm associated with pancreatitis. AJR Am J Roentgenol 1997; 169:259, 262-3.

8. Moore SW, Guida PM, Schumacher HW. Splenic artery aneurysm. Bull Soc Int Chir 1970; 29:210-8.

9. Arepally A, Dagli M, Hofmann LV, et al. Treatment of splenic artery aneurysm with use of a stent-graft. J Vasc Interv Radiol 2002; 13:631-3.

10. Dave SP, Reis ED, Hossain A, et al. Splenic artery aneurysm in the 1990s. Ann Vasc Surg 2000; 14:223-9.

11. Cappellini MD, Fiorelli G. Glucose-6-phosphate dehydrogenase deficiency. Lancet 2008; 371:64-74.

12. Glader BE. Glucose-6-phosphate dehydrogenase deficiency and related disorders of hexose monophosphate shunt and glutathione metabolism. In:Wintrobe's Clinical Hematology. 10th ed. Baltimore: Williams \& Wilkins; 2008:1176-1190.

13. Hundsdoefer P, Vetter B, Kulozuik AE. Chronic haemolytic anaemia and glucose-6-phosphate dehydrogenase deficiency. Case report and review of the literature. Acta Haematol 2002; 108:102-5.

14. Hamilton JW, Jones FG, McMullin MF. Glucose-6-phosphate dehydrogenase Guadalajara-a case of chronic non-spherocyctic haemolytic anaemia responding to splenectomy and the role of splenectomy in this disorder. Hematology 2004; 9:307-9. 